\title{
Investigating the interactions of the enantiomers of phenylglycine with nanopores of ZSM-5 zeolite
}

\author{
NAFISEH FARHADIAN ${ }^{\mathrm{a}, *}$, KOUROSH MALEK $^{\mathrm{b}}$, MOJTABA SHARIATY-NIASSAR $^{\mathrm{c}}$ and \\ ALI MAGHARI ${ }^{\mathrm{d}}$ \\ ${ }^{a}$ Chemical Engineering Department, Engineering Faculty, Ferdowsi University of Mashhad, Mashhad, Iran \\ ${ }^{\mathrm{b}}$ Department of Chemistry, Simon Fraser University, Burnaby, BC, Canada \\ ${ }^{\mathrm{c}}$ School of Chemical Engineering- University College of Engineering-University of Tehran, Tehran, Iran \\ ${ }^{\mathrm{d}}$ School of Chemistry- University College of Science-University of Tehran, Tehran, Iran \\ e-mail: na.farhadian@gmail.com
}

MS received 22 April 2013; revised 3 January 2014; accepted 6 January 2014

\begin{abstract}
In this study, molecular dynamics simulation has been used to investigate the interactions of both chiral forms of phenylglycine amino acid (R- and S-isomers) with micropores of ZSM5-zeolite. Calculated results show that phenylglycine molecules interact with zeolite surface by electrostatic interaction of their positively charged ammonium group and negatively charged carboxylic group. This leads to the formation of two hetero hydrogen bonds between amino groups and oxygen of zeolite framework and also one hydrogen bond between the carboxylic groups and the zeolite surface. Further analyses show that S-isomers have stronger interactions with zeolite surface in comparison to R-isomers. So, movement, radius of gyration and angle of orientation of S-isomers inside nanopores are decreased, while R-isomers interact more strongly with each other. However, both chiral forms have diffusive behaviour along the pores with the self diffusion coefficient of about two orders of magnitude less than that in free water.
\end{abstract}

Keywords. Nanostructure; zeolite; chirality; amino acid; molecular dynamics simulation.

\section{Introduction}

Domestic wastewaters contain the variety of organic contaminants such as pharmaceuticals and personal care products. ${ }^{1-3}$ Pharmaceuticals and antibiotics such as ampicillin, cephalexin, cephaclor, ciprofloxacin, levofloxacin and sulphamethoxazole are the most abundant residual drugs in surface waters. ${ }^{4}$ Most of these compounds undergo both incomplete removal in wastewater treatment plants and slow natural degradation. There is a particular concern about antibiotics because of their presence in natural water contributes to the spread of antibiotic resistance in microorganisms. ${ }^{5,6}$ Several methods have been reported for degrading emerging organic contaminants such as nanofiltration, adsorption, reverse osmosis, ozonation and chemical oxidation. ${ }^{7-12}$ In adsorption technology, inorganic adsorbents are often employed due to their stability toward the radioactive and thermal treatments which are necessary to induce the complete

*For correspondence degradation of adsorbates and, therefore, the regeneration of exhausted adsorbents. Among inorganic adsorbents, it has been found that zeolites are efficient in removing small organic compounds, ${ }^{13-16}$ specially amino acids ${ }^{17,18}$ from the environment. Zeolites are crystalline, microporous solids containing cavities and channels of molecular dimensions, typically $3-10 \AA$ in diameter. ${ }^{19}$ They have framework structures that are formally constructed from $\mathrm{TO}_{4}$ tetrahedra that share vertices, where $\mathrm{T}$ is a tetrahedrally-coordinated silicon or aluminum atom.

There have been some experimental ${ }^{20-23}$ and some theoretical studies ${ }^{24-26}$ which have investigated the interactions of biological molecules such as amino acids, peptides, drugs, and proteins with the surfaces of inorganic materials such as zeolites. Despite the existence of these studies, some of the fundamentals which control the interactions of biological molecules such as amino acids with zeolites are still unknown. In addition to advanced experimental techniques, versatile computational tools are generally needed to correlate transport properties of the pollutant from water. Molecular simulations have been playing an important role in providing atomistic/molecular pictures that would 
otherwise be experimentally intractable or impossible. Among molecular simulation methods, molecular dynamics (MD) simulation is a useful tool for understanding the molecular behaviour and interactions within the nanocavity of the pore network.

In this study, the interaction properties of chiral forms of phenylglycine (PHN) molecule as a chiral amino acid with ZSM-5 zeolite framework have been studied using molecular dynamics simulation method. PHN is one of the simplest chiral synthesized amino acids, with high chirality which is a good choice for molecular calculations. As it is the active side chain of some antibiotic molecules, investigating its interactions with zeolite pores can generate effective information about antibiotics separation. Some analyses such as radial distribution functions (RDF), hydrogen bond formation, atomic charge density distribution, radius gyration and molecule orientation of chiral forms of PHN molecules inside zeolite pores have been calculated. Finally, diffusion properties of this chiral amino acid inside nanopores have been discussed.

\section{Experimental}

\subsection{ZSM-5 zeolite structure}

In this study, ZSM-5 zeolite is applied with silicon and oxygen atoms which are placed according this formula: $\mathrm{SiO}_{4}$. ZSM-5 zeolite has a channel system with two sets of interconnected ring pores: 10 and 5 which form zigzag and straight channels. Thus it exhibits a 3-dimensional pore structure, in which the two channels meet in a relatively open intersection region. Figure 1 shows the applied structure of ZSM-5 zeolite in this study. The main pores are along $x$ and $y$ axes in the zigzag and straight forms, respectively. Moreover, there are two types of the pore size along $y$ axis with the averaged diameter of about 3 and $5.5 \AA$, respectively and there is one pore size along $x$ axis by the approximate diameter of $2.8 \AA$.

\subsection{PHN amino acid structure}

Phenylglycine is a synthesized alpha amino acid with the chemical formula $\mathrm{C}_{6} \mathrm{H}_{5} \mathrm{CH}\left(\mathrm{NH}_{2}\right) \mathrm{COOH}$. Similar to the natural amino acids, it contains both amino and acidic carboxylic groups. It is the side chain of some antibiotic molecules such as ampicillin, cefalexin and cefaclor (table 1). PHN is a chiral molecule. It has a zwitterion form at $\mathrm{pH}$ equals to the isoelectric point with a protonated amino cation and a carboxylate. Therefore, in this study, interactions of PHN molecule in its neutral and zwitterionic form inside nanopores of ZSM-5 zeolite are considered. In comparison to the natural amino acids, PHN is a big amino acid with a hydrophobic side chain such as phenylalanine. The length and width of this molecule are approximately 6 and $4 \AA$, respectively. Figure 2 shows the chemical structure of both chiral forms of PHN amino acid. As figure 2 shows the chemical structures of both chiral forms of PHN molecules are the same. Also, they have the same atomic Millikan charges. The only difference between R- and S-isomers of PHN molecule is their spatial structure. This property produces different interactions between these isomers with the environment.

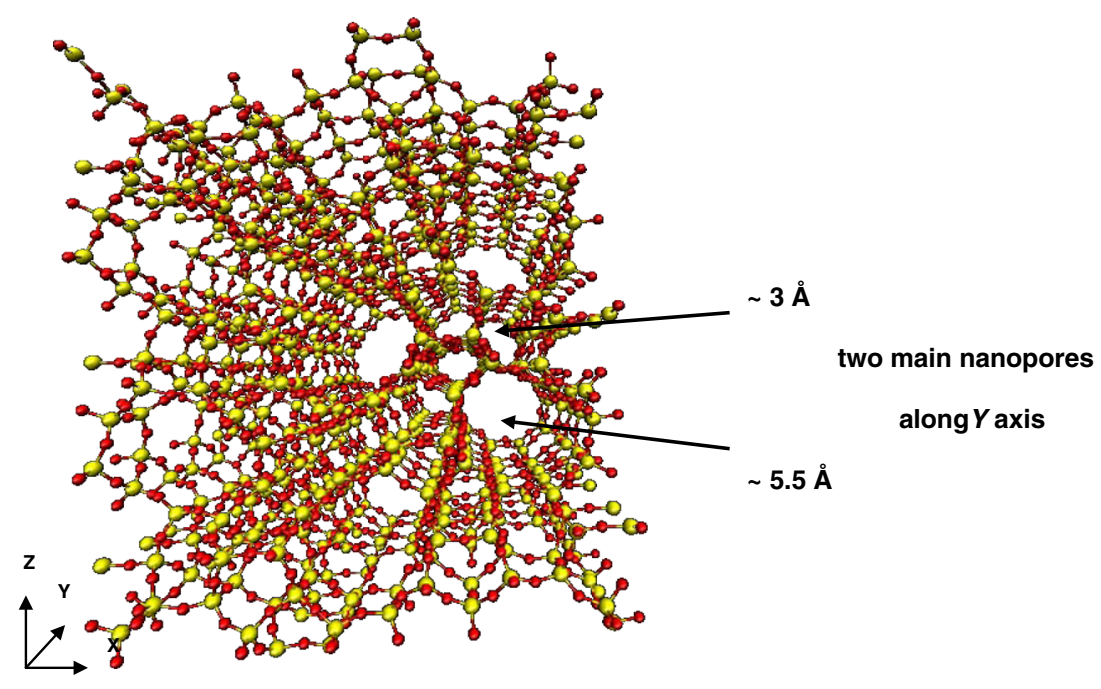

Figure 1. ZSM5-zeolite structure with the main pores along $y$ axis. 
Table 1. The structures and chemical characteristics of the pharmaceuticals under investigation.

\begin{tabular}{|c|c|c|c|}
\hline Molecule name & Chemical formula & Molecular weight $\left(\mathrm{g} \cdot \mathrm{mol}^{-1}\right)$ & Structure \\
\hline Ampicillin & $\mathrm{C} 16 \mathrm{H} 19 \mathrm{~N} 3 \mathrm{O} 4 \mathrm{~S}$ & 349.41 & \\
\hline Cefalexin & $\mathrm{C} 16 \mathrm{H} 17 \mathrm{~N} 3 \mathrm{O} 4 \mathrm{~S}$ & 347.39 & \\
\hline Cefaclor & $\mathrm{C} 15 \mathrm{H} 14 \mathrm{ClN} 3 \mathrm{O} 4 \mathrm{~S}$ & 367.808 & \\
\hline Phenylalanine & $\mathrm{C} 9 \mathrm{H} 11 \mathrm{NO} 2$ & 65.19 & \\
\hline D-alpha-phenylglycine & $\mathrm{C} 8 \mathrm{H} 9 \mathrm{NO} 2$ & 151.163 & \\
\hline
\end{tabular}

Also, there is not any inter-conversion between these two chiral forms during the MD simulation.

\subsection{Simulation set-up}

Molecular dynamics simulation method is applied to investigate the interactions of both chiral forms of PHN amino acid with ZSM5-zeolite. Following Kiselev and co-workers, ${ }^{27}$ the zeolite is modelled as a rigid crystal. This allows the use of interpolation techniques to determine the interactions of PHN amino acid with the zeolite and avoids having to consider all zeolite atoms. ${ }^{28}$ The system is consisted of a network of zeolite with
2082 atoms of $\mathrm{Si}$ and $\mathrm{O}$ and $78 \mathrm{PHN}$ molecules to prepare a $0.5 \mathrm{M}$ aqueous mixture in the simulation box with the size of $3.150 \mathrm{~nm} \times 6.000 \mathrm{~nm} \times 3.900 \mathrm{~nm}$. The equal numbers of S- and R- forms of PHN molecules are manually positioned at two sides of the zeolite along the $y$ axis to produce a uniform structure towards various chiral forms of PHN molecules. The simulation box has been fully hydrated with water molecules. SPC model is used to correlate the solvent interactions ${ }^{29}$ and Gromos 96 force field is applied for PHN and zeolite network. This force field includes any necessary expressions for describing bond-stretching, bond-bending and torsional potentials, as well as Lennard-Jones and 


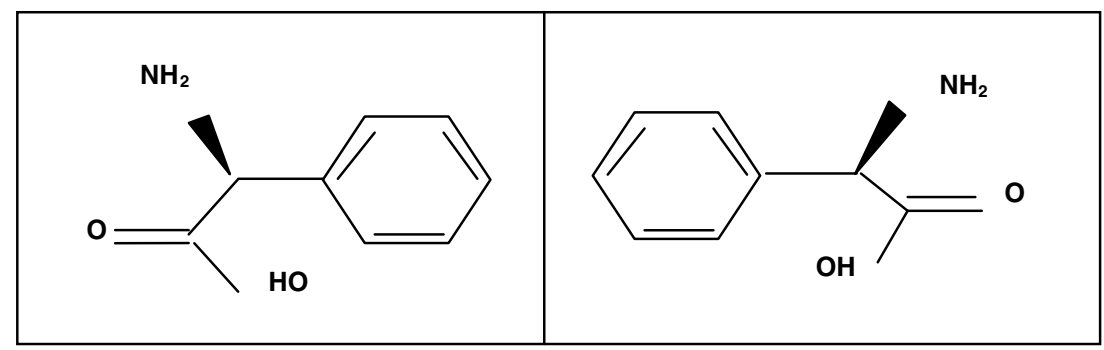

(a)

(b)

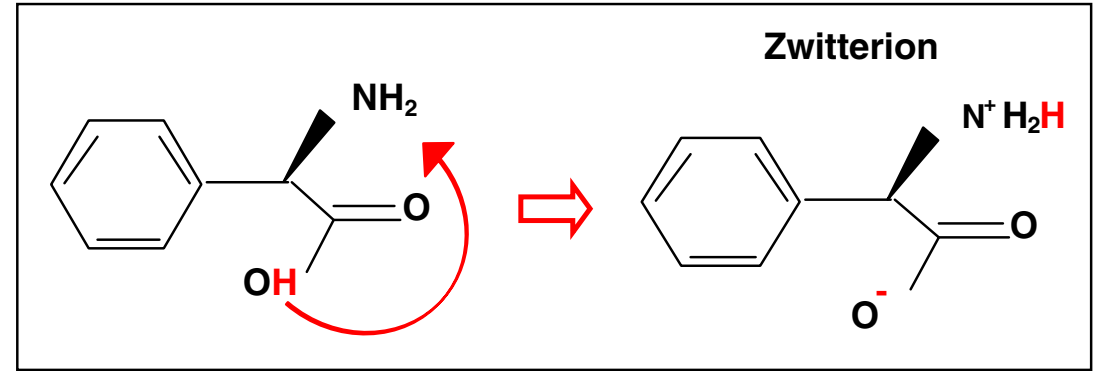

(c)

Figure 2. Schematic of chiral forms of phenylglycine molecule (a) S-form, (b) R-form and (c) zwitterion formation.

Table 2. The bonded and non-bonded force field parameters for PHN and zeolite atoms.

\begin{tabular}{lcc}
\hline & Bonded parameters & \\
\hline Bond & $\mathrm{K}\left(\mathrm{KJ}_{\mathrm{m}} \mathrm{mol}^{-1}\right)$ & $\mathrm{r}_{0}(\mathrm{~nm})$ \\
N(chiral)-CH & 376560 & 0.147 \\
N(chiral)-H & 374468 & 0.100 \\
CH-C(aromatic) & 418400 & 0.139 \\
CH-CH (aromatic) & 418400 & 0.139 \\
C (aromatic)-CH & 334720 & 0.153 \\
CH-C (carboxylic) & 334720 & 0.153 \\
C (carboxylic)-O(hydroxyl) & 418400 & 0.125 \\
C (carboxylic)-O(carbonyl) & 418400 & 0.125 \\
Si-O (zeolite) & 418400 & 0.159 \\
& & $\theta_{0}\left({ }^{\circ}\right)$ \\
Angle & $\mathrm{K}\left(\mathrm{KJ} . \mathrm{mol}^{-1}\right)$ & 120 \\
C(aromatic)-CH-CH & 418.4 & 120 \\
CH-C(aromatic)-CH & 418.4 & 109.5 \\
C(aromatic)-CH-N (chiral) & 460 & 120 \\
O(carboxyl)-CH-N (chiral) & 418.4 & 109.5 \\
C(aromatic)-CH-C(carboxyl) & 460 & 117 \\
CH-C(carboxylic)-O(hydroxyl) & 502 & 117 \\
O(hydroxyl)-C(carboxyl)-O(carbonyl) & 502 & 109.5 \\
O-Si-O (zeolite) & 418.4 & $\Psi_{0}\left(^{\circ}\right)$ \\
Dihedral & & 0 \\
C(aromatic)-CH-CH-CH & $\mathrm{K}\left(\mathrm{KJ} \cdot \mathrm{mol}^{-1}\right)$ & 0 \\
C(carboxyl)-CH-O(carbonyl)-O(hydroxyl) & 1673 & 180 \\
O-Si-O-Si (zeolite) & 1673 & $\sigma(\mathrm{nm})$ \\
& 5.00 & 0.339 \\
Atom & $\varepsilon / \mathrm{k}_{\mathrm{B}}(\mathrm{K})$ & 0.303 \\
Si & 294.5 & 0.34 \\
O & 80.5 & \\
C & 28.1 & \\
\hline
\end{tabular}




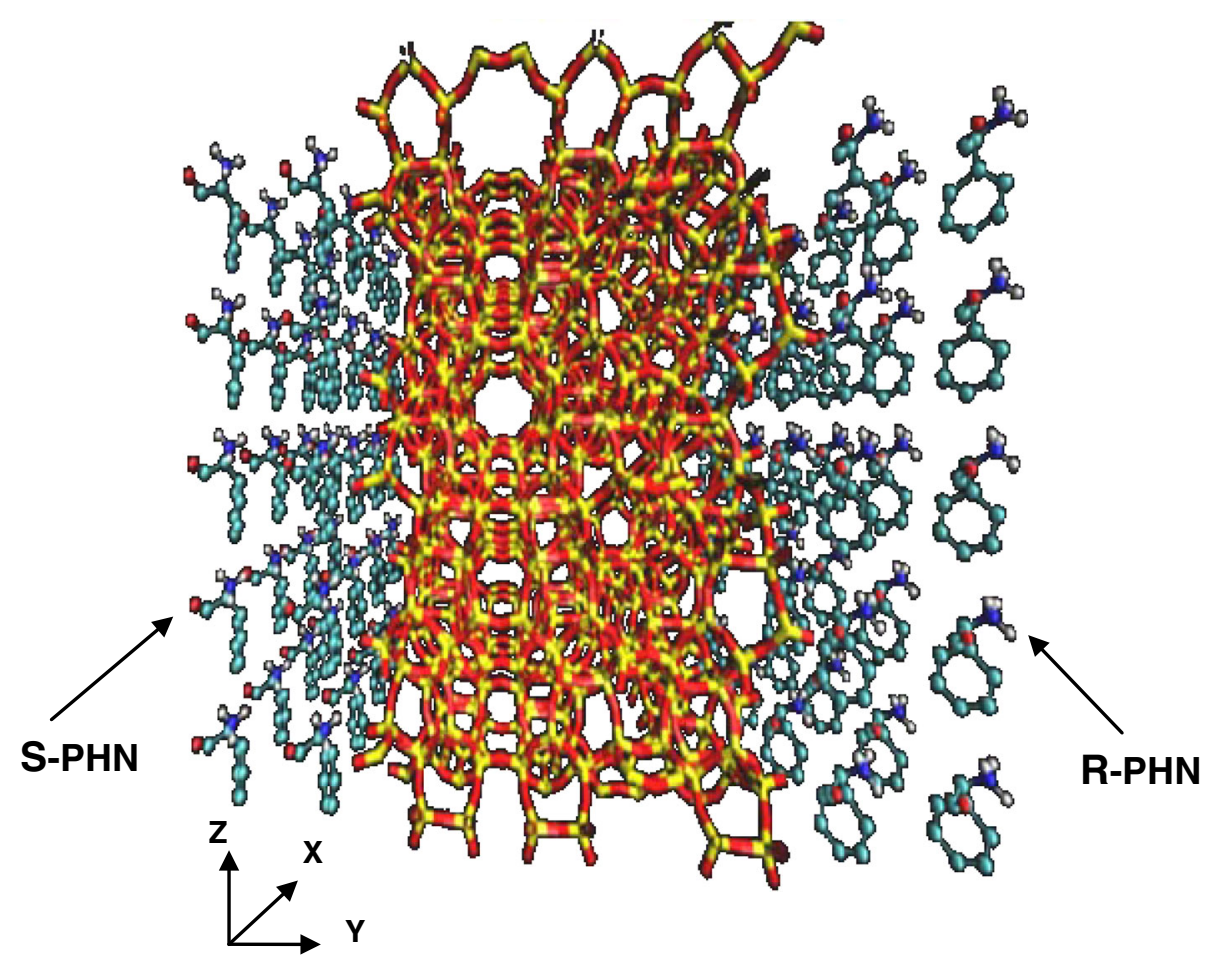

Figure 3. Schematic of the initial simulated system without water molecules.

partial charge parameters for the atoms of the sorbate molecules that is expressed as equation $1:^{30}$

$$
\begin{aligned}
E= & \sum_{\text {bond }} \frac{1}{2} K_{r}\left(r-r_{0}\right)^{2}+\sum_{\text {angle }} \frac{1}{2} K_{\theta}\left(\theta-\theta_{0}\right)^{2} \\
& +\sum_{\text {torsion }} \frac{K_{\varphi}}{2}\left[1+\cos \left(n \varphi-\varphi_{0}\right)\right] \\
& +\sum_{i<j}\left\{\left[\left(\frac{\sigma_{i j}}{r_{i j}}\right)^{12}-\left(\frac{\sigma_{i j}}{r_{i j}}\right)^{6}\right]+\frac{1}{4 \pi \varepsilon_{0}} \frac{q_{i} q_{j}}{R_{i j}}\right\},
\end{aligned}
$$

where $r, \theta$ and $\varphi$ are the bond length, bond angle and torsion angle, respectively. The subscript 0 represents their equilibrium values. Lennard-Jones and coulomb potential are used as the non-bonding atom-atom interaction where $r_{i j}$ is the distance between the nonbonding atoms, $\sigma_{i j}$ and $\varepsilon_{i j}$ are the Lennard-Jones size and energy parameters, $q_{i}$ and $q_{j}$ are the atomic point charges, respectively. The applied force field parameters for PHN and zeolite have been presented in table 2 . Canonical ensemble $(N V T)$ and the periodic boundary condition are used for the simulation. Simulation has been performed at constant temperature of $300 \mathrm{~K}$. To maintain a constant temperature, Berendsen coupling algorithm is applied. The electrostatic interactions in the simulations are calculated using the particle-mesh Ewald (PME) method. ${ }^{31}$ At first, system is energy minimized for 200 ps and after that MD simulation is performed for 9 ns using a time step of 2 fs. Equilibration state is monitored by calculating the total energy of the system versus simulation time. Simulation is carried out using GROMACS software ${ }^{32}$ and visualizations are done by VMD V.1.8.6 commercial package. ${ }^{33}$ The configuration of the simulated system without water molecules for better understanding is shown in figure 3.

\section{Results and discussion}

At first, to investigate the interactions of PHN molecules with the zeolite surface, the radial distribution function (RDF) of two active sites of PHN molecules (amino and carboxylic groups) and zeolite surface is calculated during the simulation time. RDF describes how the atomic density varies as a function of the distance from one particular atom. Figure 4 shows the RDF of amino and carboxylic groups of both chiral forms of PHN molecules toward oxygen 

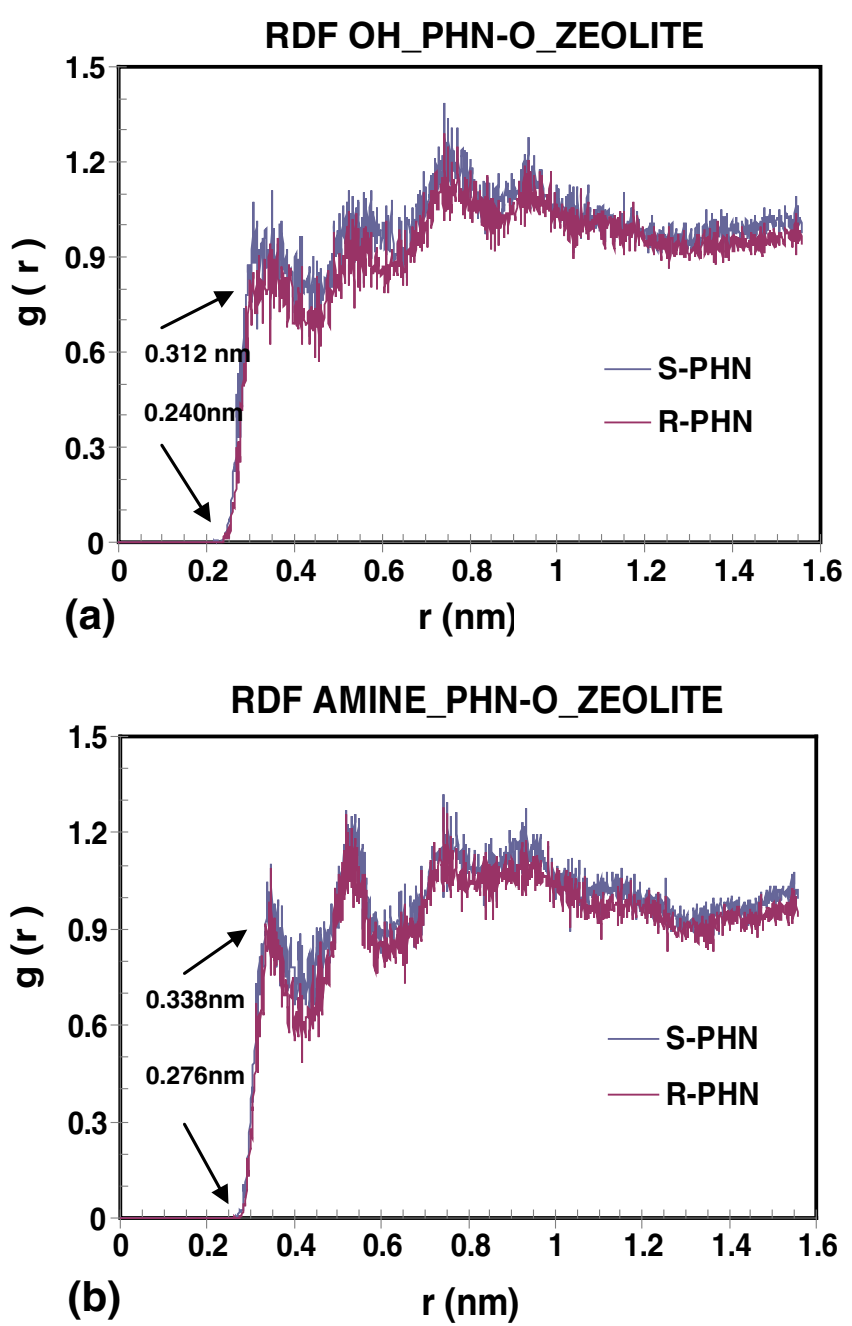

Figure 4. Radial distribution function of carboxylic (a) and amino (b) groups of PHN with zeolite framework. atoms of zeolite. Results show that both amino and carboxylic groups of PHN molecule interact with the zeolite framework but hydroxyl side chain of carboxylic group has stronger interactions with the zeolite surface due to its closer distance toward the oxygen atom of the zeolite surface. Further analysis such as hydrogen bond formation shows that two hetero-hydrogen bonds $(\mathrm{N}-\mathrm{H} . . \mathrm{O})$ between the amino group and the zeolite framework and one hydrogen bond $(\mathrm{O}-\mathrm{H}$. . .O) between hydroxyl of carboxylic group and the zeolite framework are formed. The $\mathrm{N}-\mathrm{H}$. . .O hydrogen bond distance is computed to be $1.73 \AA$ and $1.69 \AA$ while the O-H. . . O hydrogen bond distance is $1.63 \AA$ (figure 5). The hydroxyl group interaction with zeolite surface in zwitterion form is based on the formation of a complex by transferring the hydrogen atom of amino group near the zeolite oxygen atom and formation of new hydrogen bonds (figure 5b). Indeed, two different peaks can be observed in $\mathrm{N}-\mathrm{H}$ bond distribution profile (figure 6). The first peak can be observed at distance $0.1 \mathrm{~nm}$ that is the bond length of $\mathrm{N}-\mathrm{H}$ in amino group $\left(\mathrm{NH}_{3}^{+}\right)$and another is at the distance of $0.164 \mathrm{~nm}$ which is related to the hydrogen transferring in amino group toward oxygen atom of hydroxyl group $\left(\mathrm{NH}_{2} \ldots \mathrm{H}^{+} \ldots{ }^{-} \mathrm{O}-\mathrm{C}=\mathrm{O}\right)$. After these bond formations, the carbonyl bond length $(\mathrm{C}=\mathrm{O})$ is lengthened from $1.21 \AA$ to $1.23 \AA$. Also, the angle between $\mathrm{N}-\mathrm{H}$ groups and $\mathrm{C}-\mathrm{C}-\mathrm{O}$ decreases from $109.5^{\circ}$ and $121^{\circ}$ to $107.577^{\circ}$ and $120.323^{\circ}$, respectively and mutually the angle between $\mathrm{C}-\mathrm{C}-\mathrm{C}$ and $\mathrm{C}-\mathrm{C}-\mathrm{N}$ increases from $109.5^{\circ}$ to about $112^{\circ}$. These changes between bond length and bond angles at both isolated and adsorbed states are listed in table 3.

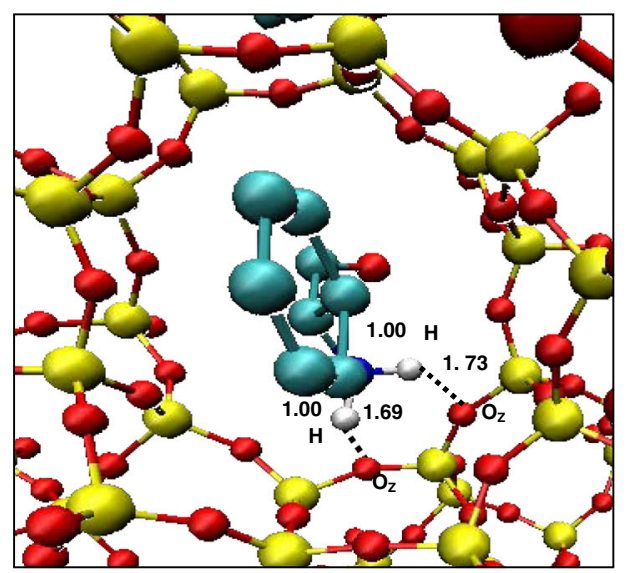

(a)

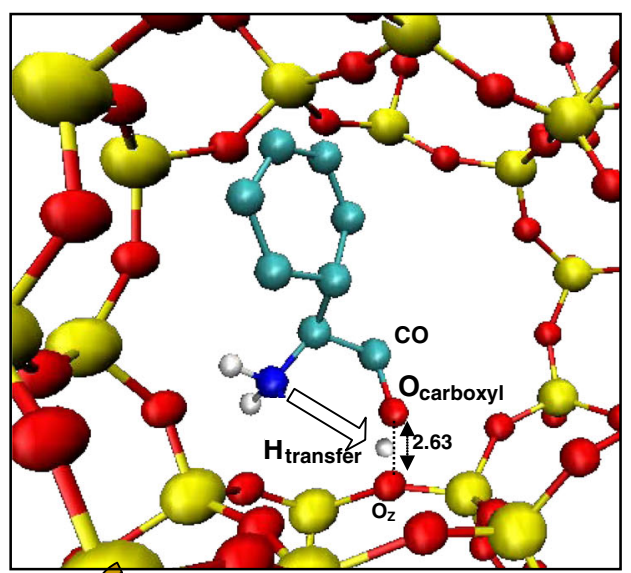

(b)

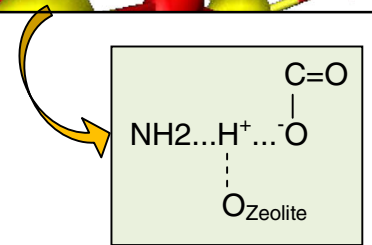

Figure 5. Two hetero-hydrogen bonds between (N-H. . . O) (a) and one hydrogen bond between (O-H. . O) (b). 


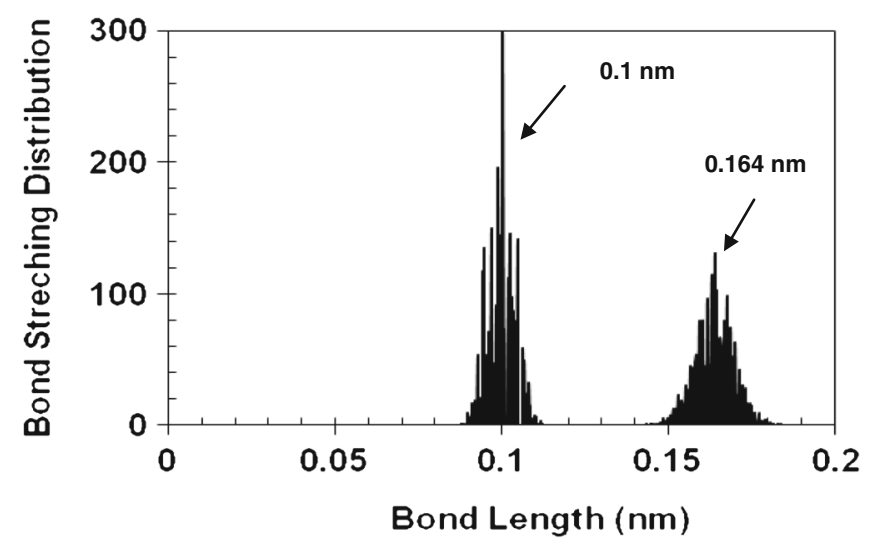

Figure 6. Bond length distribution of $\mathrm{N}-\mathrm{H}$ in $\mathrm{PHN}$ molecule.

Moreover, hydrogen bond analysis shows that chiral forms of PHN molecules interact to each other inside nanopores of zeolite. In that case, amino groups play as both donor and acceptor of hydrogen. The number of hydrogen bond between PHN molecules and zeolite surface decreases according to this order: (R-isomer with $\mathrm{S}$-isomer $)>(\mathrm{S}$-isomer with zeolite $)>$ (R-isomer with zeolite) $>$ (R-isomer with R-isomer $)>$ ( $\mathrm{S}$-isomer with $\mathrm{S}$-isomer). This observation indicates that PHN molecules are able to pass each other inside pores. Hence, rapid filling of the zeolite pores with PHN molecule occurs. It has an important effect on PHN alignment inside pores. PHN molecules orient their polar head to the channel and also to the polar head of another PHN molecule. In the solvent environment, this causes more hydrophobic interactions between nonpolar side chains of adsorbed amino acids with water molecules. This speeds up the separation of amino acids from aqueous solutions more effectively. This observation is in agreement with some experimental work ${ }^{34}$ and also theoretical calculations such as glycine natural amino acid transportation on the silica surfaces. ${ }^{35}$ However, these different interactions between PHN molecules and zeolite surface produce various atomic charge density inside zeolite pores (figure 7). Here, calculated charge density is the electrostatic potential across the box. This electrostatic potential has been calculated by the first summation of the charges per slice and then integrating twice of this charge distribution. ${ }^{31}$ From figure 7, it can be found that the maximum charge density is related to the amine and hydroxyle groupes of S-isomers along $y$-axis. This observation indicates that $\mathrm{S}$-isomers have stronger interactions with zeolite network in comparison to R-isomers. This is in agreement with the number of hydrogen bond discussed above. This causes less movement and orientation of S-isomers along pores. Figure 8 shows the coordination and radius gyration of both chiral forms of PHN molecules along zeolite pores. Moreover, to investigate the orientational order of the chiral forms of PHN molecules inside zeolite channel, the first and second order Legendre polynomial are calculated. The first order Legendre polynomial $\left(P_{1}(\cos \theta)=\cos \theta\right)$ shows the average value of the dipole moment distribution and the second order Legendre polynomial $\left(P_{2}(\cos \theta)=1 / 2\left(3 \cos ^{2} \theta-1\right)\right)$ provides a measure of deviation from this average. From figure 9 , it can be concluded that both PHN isomers have nearly

Table 3. The bond distance and angle parametersof PHN - ZSM5 zeolite $\left(\AA\right.$, $\left.^{\circ}\right)$ for isolated and adsorbed structure complexes.

\begin{tabular}{|c|c|c|c|}
\hline & \multirow[b]{2}{*}{ Isolated } & \multicolumn{2}{|c|}{ Adsorbed } \\
\hline & & Amino group & Carboxylic group \\
\hline \multicolumn{4}{|l|}{ Bond distance $(\AA)$} \\
\hline $\mathrm{C}=\mathrm{O}$ & 1.210 & - & 1.230 \\
\hline $\mathrm{N}-\mathrm{H} 1$ & 1.000 & 1.000 & - \\
\hline $\mathrm{N}-\mathrm{H} 2$ & 1.000 & 1.000 & - \\
\hline N-H3(transfer) & 1.000 & 1.640 & \\
\hline \multicolumn{4}{|l|}{ Angle $\left(^{\circ}\right)$} \\
\hline $\mathrm{C}-\mathrm{C}-\mathrm{N}$ & 109.500 & 112.687 & - \\
\hline $\mathrm{C}-\mathrm{N}-\mathrm{H}$ & 109.500 & 107.577 & - \\
\hline $\mathrm{C}-\mathrm{C}-\mathrm{C}$ & 109.500 & - & 112.323 \\
\hline $\mathrm{C}-\mathrm{C}=\mathrm{O}$ & 121.000 & - & 120.512 \\
\hline \multicolumn{4}{|l|}{ Hydrogen bond distance $(\AA)$} \\
\hline $\mathrm{N}-\mathrm{H} 1 \ldots \mathrm{Oz}$ & - & 2.690 & - \\
\hline $\mathrm{N}-\mathrm{H} 2 \ldots \mathrm{Oz}$ & & 2.730 & \\
\hline C- $\mathrm{O}_{\text {hydroxyle }} \ldots \mathrm{H}_{3 \text { (transfer) }} \ldots \mathrm{Oz}$ & - & - & 2.630 \\
\hline
\end{tabular}



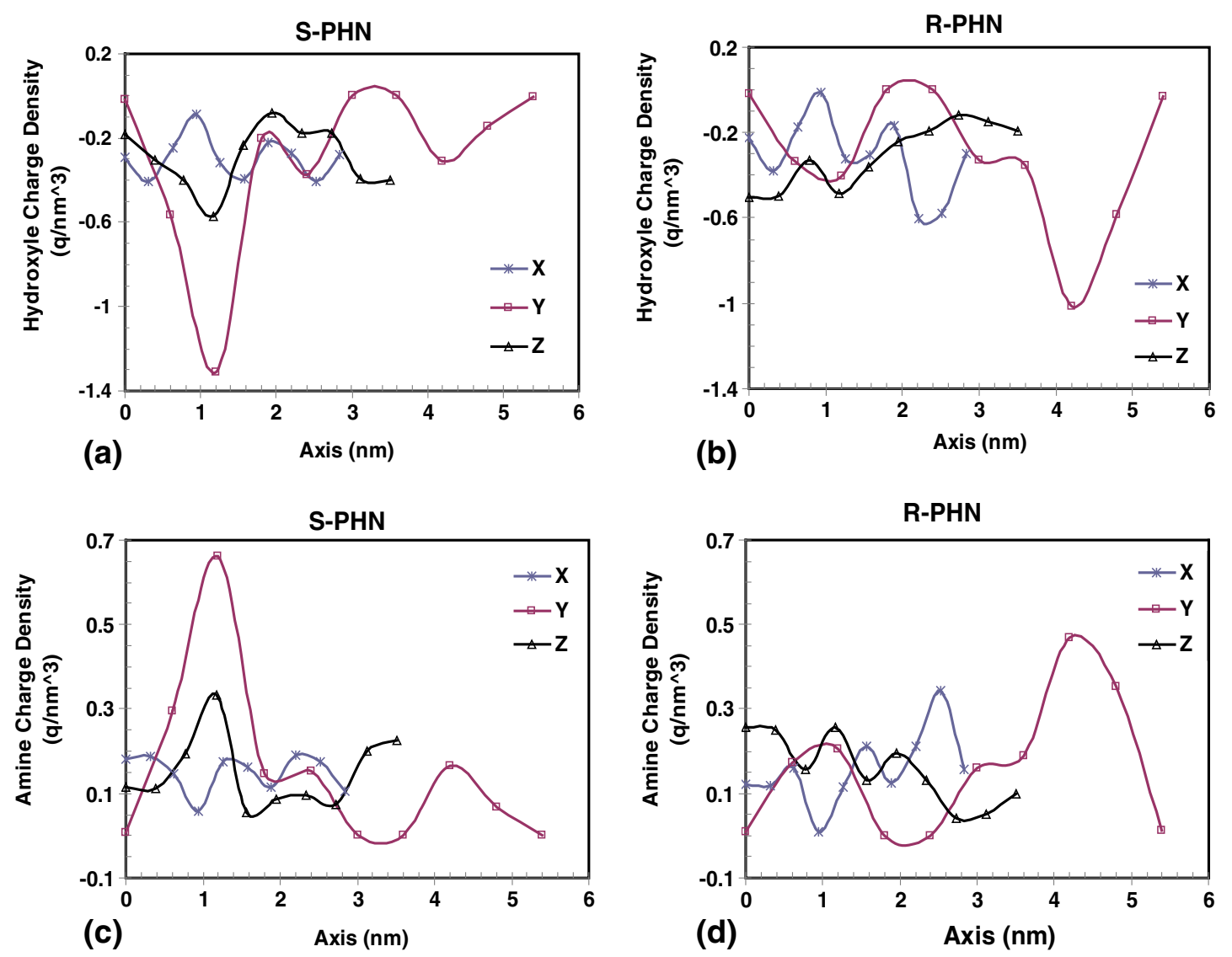

Figure 7. Atomic charge density along different axes for hydroxyl (a,b) and amino (c,d) groups of chiral forms.

the same dipoles pointed upward $(P 1)$, but $\mathrm{S}$-isomers prefer an orientation in either direction because of the $P 2$ curve that is closer to +1 . This overview indicates that $\mathrm{S}$-isomers are located inside pores more regularly with less fluctuation rather than R-isomers.

Finally, self diffusion coefficient of chiral forms of PHN molecules in the zeolite framework is calculated by mean square displacement (MSD) analysis. Figure 10 shows the MSD of the centre of mass of chiral forms of PHN molecules as a function of time. The self diffusion coefficient $(D)$, is related to the slope of the mean square displacement of solute molecules after time that their motion is equilibrated by the Einstein relation, ${ }^{36}$ which in $d$ dimension is:

$$
D=\lim _{\Delta t \rightarrow \infty} \frac{\left(|\Delta \bar{r}|^{2}\right)_{t 0}}{2 d \Delta t}
$$

where $\left(|\Delta \bar{r}|^{2}\right)_{t 0}$ is the MSD of solute molecules during the time $\Delta t$, averaged over the ensemble of molecules in a $d$ dimensional space, from the moment $t_{0}$ that their motion is equilibrated. Calculations show that system reaches to the equilibrium after $1 \mathrm{~ns}$, so this time is selected as $t_{0}$ and MSD curve versus time is plotted after this time. Figure 10 shows that the MSD curve of PHN molecules versus time during $1 \mathrm{~ns}<\mathrm{t}<9$ ns is linear $\left(\mathrm{R}^{2}>0.98\right)$. This observation predict a diffusive behaviour of PHN molecules (MSD is proportional to $t$ and the slope of the log-log curve as shown in inset diagram approximates to one $\left.\left(\mathrm{R}^{2}>0.98\right)\right)$. So, Einstein equation can be applied to predict the self diffusion coefficient. Use of the Einstein equation for determination of $D$ requires a linear dependence of the MSD with time. Applying figure 10, it can be concluded that the slope of the MSD curve versus time for R-isomers is more than that for S-isomers. This confirms that the self diffusion coefficient for R-isomers is more than that for S-isomers. The averaged calculated diffusion coefficient of R-isomers along the zeolite pores during $1 \mathrm{~ns}$ $<\mathrm{t}<9$ ns is equal to $1.56 \times 10^{-11} \mathrm{~m}^{2} . \mathrm{s}^{-1}$, while this value decreases to $1.15 \times 10^{-11} \mathrm{~m}^{2} . \mathrm{s}^{-1}$ for $\mathrm{S}$-isomers. 

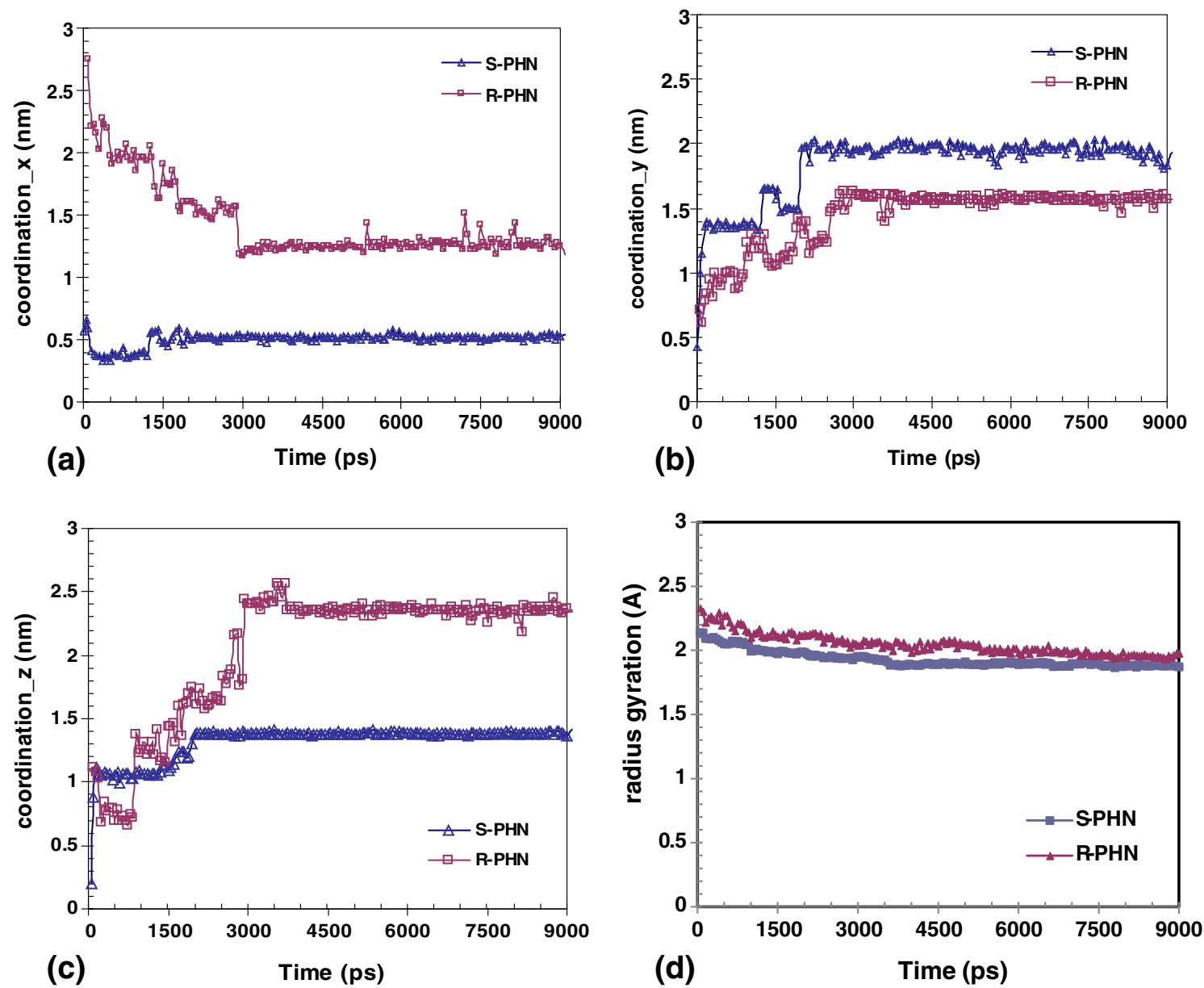

Figure 8. R- and S-coordination along $x(\mathbf{a}), y(\mathbf{b})$ and $z$ (c) axes and radius gyration of chiral forms of PHN molecules inside pores $(\mathbf{d})$.

Both of these calculated diffusion coefficients of PHN molecules inside zeolite nanopores are two orders of magnitude less than those in free water. ${ }^{37}$ As previously discussed, R-isomers have the biggest interactions to each other which cause their effective passage inside pores with higher diffusion coefficient, but S-isomers
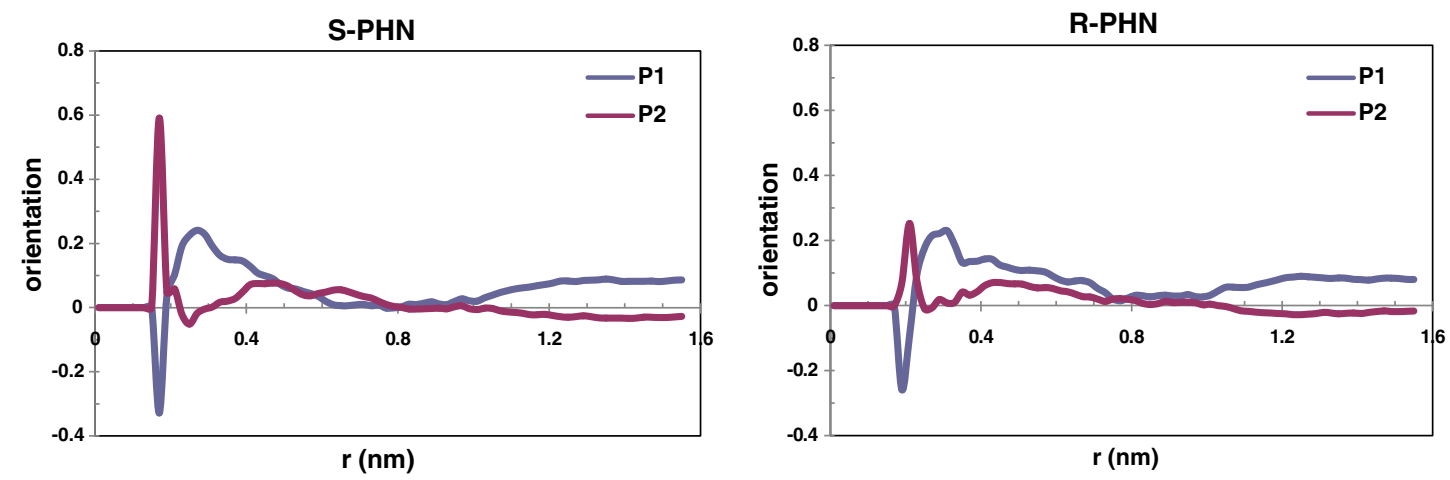

Figure 9. The first and second order Legendre polynomial of the S-isomer (a) and R-isomer (b) of PHN molecules inside zeolite. 


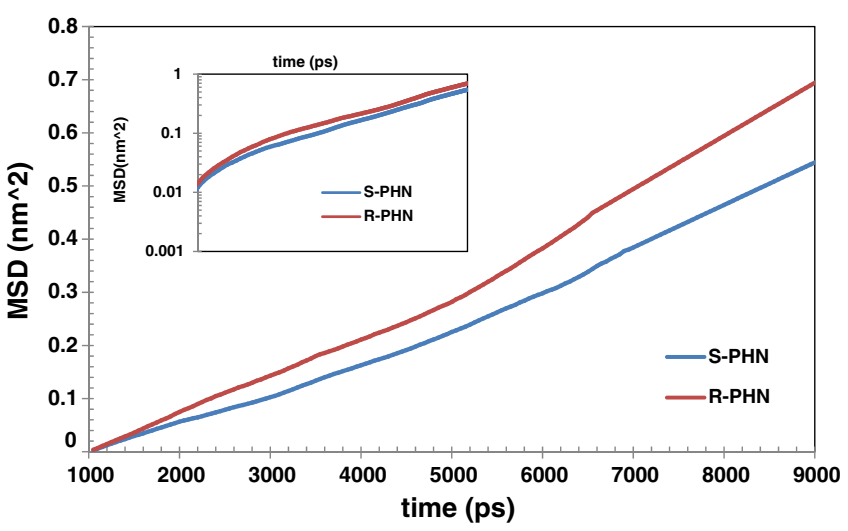

Figure 10. MSD of PHN molecules for R- and S-isomers versus time, inset diagram shows the logarithmic scale of MSD curve versus time.

have less interactions to each other resulting less fluctuations inside pores with lower diffusion coefficient value.

\section{Conclusion}

Detailed understanding of transport process in zeolites requires further of the way in which molecules fit into the pores of such zeolites. Computational methods are now providing extraordinary insight into these phenomenons. Indeed at this work, interactions of chiral forms of PHN molecules as high polar chiral amino acid with ZSM5-zeolite have been studied using molecular dynamics simulation. Results show that PHN molecules interact with zeolite surface by the formation of hydrogen bond between their amino and carboxylic groups and zeolite surface. Further analysis show that Sisomers have stronger interactions with zeolite surface which creates less gyration and angle orientation inside pores. But R-isomers have strong interactions with each other which leads to rapid passage inside pores. This behaviour inside pores leads to greater diffusion coefficient for R-isomers than for S-isomers. PHN diffusion coefficient inside zeolite pores is two orders of magnitude less than that in free water.

\section{References}

1. Löffler D, Römbke J, Meller M and Ternes T A 2005 Environ. Sci. Technol. 395209

2. Reemtsma T, Weiss S, Mueller J, Petrovic M, González S, Barcelo D, Ventura F and Knepper T P 2006 Environ. Sci. Technol. 405451

3. Loraine G A and Pettigrove M E 2006 Environ. Sci. Technol. 40687
4. Figueroa R A, Leonard A and MacKay A A 2004 Environ. Sci. Technol. 38476

5. Lienert J, Güdel K and Escher B I 2007 Environ. Sci. Technol. 414471

6. Knapp C W, Dolfing J, Ehlert P A I and Graham D W 2010 Environ. Sci. Technol. 44580.

7. Conn K E, Barber L B, Brown G K and Siegrist R L 2006 Environ. Sci. Technol. 407358

8. Ternes T A, Meisenheimer M, McDowell D, Sacher F, Brauch H J, Haist-Gulde B, Preuss G, Wilme U and Zulei-Seibert N 2002a Environ. Sci. Technol. 363855

9. Heberer T 2002 Hydrol. J. 266175

10. Daughton C G and Jones-Lepp L 2001 American Chemical Society, (Washington DC) Symposium Series p. 791

11. Huber M M, Canonica S, Park G-Y and Gunten U 2003 Environ. Sci. Technol. 371016

12. Ternes T A, Stüber J, Herrmann N, McDowell D, Ried A, Kampmann M and Teiser B 2003 Water Res. 37(8) 1976

13. Zhao X S, Ma Q and Lu G Q 1998 Energy Fuels 121051

14. Anderson M A 2000 Environ. Sci. Technol. 34725

15. Tuan V A, Li S, Noble R D and Falconer J L 2003 Environ. Sci. Technol. 374007

16. Hung H-W and Lin T-F 2006 Hazard. J. Mater. 135210

17. Krohn J E and Tsapatsis M 2005a Langmuir 218743

18. Krohn J E and Tsapatsis M 2006b Langmuir 229350

19. van Bekkum H, Flanigen E M, Jacobs P A and Jansen J C 2001 Introduction to zeolite science and practice, (2nd ed.) (Amsterdam: Elsevier)

20. Tzvetkov G, Koller G and Zubavichus Y 2004 Langmuir 2010551

21. Meng M, Stievano L and Lambert J-F 2004 Langmuir 20914

22. Gray J J 2004 Curr. Opin. Struct. Biol. 14110

23. Hartmann M 2005 Chem. Mater. 174577

24. Aquino A J A, Tunega D and Gerzabek M H 2004 J. Phys. Chem. B 10810120

25. Rimola A, Tosoni S and Sodupe M 2006a ChemPhysChem. 7157

26. Rimola A, Sodupe M and Tosoni S 2006b Langmuir 22 6593

27. Bezus A G, Kiselev A V, Lopatkin A A and Du P Q J 1978 J. Chem. Soc. Faraday Trans. 2367

28. Smit B and Siepmann J I J 1994 J. Phys. Chem. 988442

29. Berendsen H J C, Postma J P M, von Gunsteren W F and Hermans J 1981 Intermolecular forces (Dordrecht: Reidel Publ)

30. Gunsteren W F and Berendsen J C 1990 Angew. Chem. Int. Ed. Engl. 29992

31. de Leeuw S W and Perram J W 1979 Mol. Phys. 371313

32. Lindahl E, Hess B and van der Spoel D $2001 \mathrm{~J}$. Mol. Mod. 7306

33. Humphrey W, Dalke A and Schulten K 1996 J. Mol. Graphics 14(1) 33

34. Munsch S, Hartmann M and Ernst S 2001 Chem. Commun. 191978

35. Aikens M C and Gordon M S 2006 J. Am. Chem. Soc. 12812835

36. Malek K, Odijk T and Coppens M-O 2005 Nanotechnology 16522

37. Farhadian N, Malek K, Shariaty-Niassar M and Maghari A 2011 Chem. Lett. 40 